\title{
Greek students' images of scientific researchers
}

\author{
Vasilia Christidou
}

\begin{abstract}
Public images of scientific researchers - as reflected in the popular visual culture as well as in the conceptions of the public- combine traditional stereotypic characteristics and ambivalent attitudes towards science and its people. This paper explores central aspects of the public image of the researcher in Greek students' drawings. The students participated in a drawing competition held in the context of the 'Researcher's Night 2007' realized by three research institutions at different regions of Greece. The students' drawings reveal that young people hold stereotypic and fairly traditional and outdated views of scientists and scientific activity. Research institutions are faced with the challenge of establishing a sincere and fertile dialogue with society to refute obsolete and deceiving notions and to promote the role of researchers in society.
\end{abstract}

\section{Introduction}

For more than a decade there has been wide international concern for the improvement of the level of public engagement in science -especially among young people. This concern has been expressed in different ways both within the scope of formal education, as well as through open interventions to the general public. Public engagement in science issues is related to the way in which one conceptualizes the nature of technological and scientific research and the people engaged in it.

Actions aiming at familiarizing the public with the people of research and at diffusing their accomplishments have, among others, the central objective of promoting a realistic image about the world of science and technology. Quite often, non-specialists, including students as future citizens, and potential researchers themselves, hold traditional, perplex and inaccurate perceptions about scientific research. These perceptions may actually be associated with strong skepticism and negative attitudes towards science, and with a reluctance of young people, especially in the developed countries, towards research both at the level of studies and at the level of career choices. . $^{2,3,4,5,6,7}$

According to Schummer \& Spector ${ }^{8}$ the public image of almost anything is substantially a visual image. Research interest in the public engagement in science issues has emerged simultaneously with the development of visual studies and, although the public image of science has become an important topic, the public visual image of science has not. However, science-related texts have been relying on visualization at least since medieval times.

This paper aims at revealing significant aspects of the public image of scientific researchers, as reflected in the visual images (drawings) produced by Greek students. In the following sections the public image of techno-scientific research -as outlined by previous studies- will be described, the context of the study and the main outcomes will be presented, and the relevant conclusions will be drawn.

\section{The public image of scientific research}

The image prevailing in society about scientific research constitutes a relatively stable amalgam of traditional stereotypes, which draw their historic references from the pre-scientific period (even from the $14^{\text {th }}$ century alchemists) and modern, constantly transforming perceptions about scientific and technological progress and its positive and negative effects on society and the planet. ${ }^{8,9}$ This image reveals the low level of public understanding of the professions related with science. At the same time, it 
reflects the stability of 'collective meanings' apparently related to myths and stereotypes with deep cultural roots. ${ }^{10}$

This popular image of scientific research also indicates ambivalence and a retrogression of the public between trust and mistrust towards science, between faith in progress on one hand and skepticism towards technology, or fear about its uncontrollable effects on the other. ${ }^{10}$ This contradictory image is revealed by research studies concerning both the public representation of scientists in comic strips and clipart cartoons, fiction films, or in the mass media ${ }^{8,10,11,12,13}$ and the conceptions and attitudes of the public -including students and teachers- about scientists and their activity.

The term 'images of science and scientists' mainly refers to stereotypes and caricatures of technicians wearing white coats, working in gleaming laboratories and using complex technological equipment to carry out measurements, gather data and demonstrate their hypotheses. Such stereotypic images are dominant within the general public and are promoted by the mass media. Various studies agree that such images develop quite early, even from the age of 8 , and are continually enhanced until adolescence. $^{4,22,23,24,25,26}$ These images are adopted by male and female students, with various national and cultural backgrounds, ${ }^{5,7,16,26,27,28}$ as well as by teachers. ${ }^{15,29,30,31,32}$

Scientists are mostly men, generally represented as geniuses, hard-working and attentive to the degree of obsession, surrounded by a veil of abstraction, confusion, even 'madness'. Sometimes their enthusiasm and scientific curiosity can put humanity in danger, since they possess secret knowledge and power over nature. Within this picture scientists can be 'madmen', capable of disastrous inventions and dangerous interventions on nature, or gentle gurus devoted to saving the world. ${ }^{7,14}$ Their social dexterity is rather limited: they are often presented as inept, isolated and unsociable. ${ }^{4,15}$ They are not interested in social trends, or in fashion. Their appearance, with eyeglasses, white lab coats and tangled hair is rather unattractive.

In other cases the image of scientists is dominated by positive - albeit stereotypic - characteristics: they are eccentric but harmless, as experts they know everything, they are dedicated to the extension of knowledge boundaries, ${ }^{15}$ and devote their wisdom to the service of society. They combine an optimistic faith in a better future with an unsaturated curiosity, dynamism, an aspiration for investigation, and an aptitude for exploring new routes. ${ }^{10,11}$

In any case, the prevailing image in the public field obviously implies that the scientific researcher is not 'someone like us', but constitutes the central hero of a broad mythology about the world of science and the big adventure of the conquest of knowledge.

Women are hardly present in the public image of science. ${ }^{10,16}$ The sporadic representations of women scientists mainly incorporate components of gender stereotypes rather than scientific ones. Their roles are secondary and differentiated from those of their male counterparts. In more traditional versions of this stereotype, a woman works under the supervision of a man, as a student or an assistant. In more contemporary versions, the 'only' impediment in undertaking a leading or equivalent role to that of men is a male-dominated scientific system, and frequently their 'immature', or exceedingly sentimental nature.,

The widespread stereotype of the 'mad scientist' does not apply in the case of women scientists, who generally do not contribute to the negative myths of science. ${ }^{10}$ They do not work in secret laboratories, or in controversial research programs, but remain realistic and dedicated to the general good, adding intuition and emotion to scientific research. They also embody the societal fears and reflect the public skepticism about disputable scientific issues.

Scientific research is misleadingly presented in the public sphere as a primarily practical and mechanistic activity of proof and demonstration, of an experimental and quantitative nature, collection of data, foundation of rules, certainties, and positive knowledge. ${ }^{13}$ The public adopts this conception. Moreover, studies involving students indicate that scientific research is considered -mainly by girls- as competitive, impersonal, abstract, and deprived of imagination. ${ }^{16}$

The origin and underlying cause of those stereotypic images are difficult to trace. On the one hand a 'popular visual image' of scientists has been cultivated for decades - if not centuries - and is being reproduced by the media, literature, the internet and other channels of diffusion to the public field. On the other, the media, which address the general public, also address its anticipations and experiences, by mediating images that echo the public opinion. Therefore this image is particularly strong because it is recognizable by the public. Hence, there is an essential coincidence and constant interaction and mutual amplification between the images projected by the media and those held by the public about technoscientific research and its people. ${ }^{10}$ 
This 'popular visual image' is mainly nourished by science fiction and less by actual scientific research. Therefore, while there are only few real scientists known to the general public, the corresponding fantastic heroes (like Frankenstein) are very popular. ${ }^{11,17,18,19}$ These fictional heroes determine the images of science that are (re)produced and diffused by the public media to a much larger extent than real researchers.' Obviously the self-concept and the self-image of the scientific community differ significantly from those of the public. However, scientists themselves often reproduce even the most conservative stereotypes -to which they otherwise object- in their self-representations, thus participating in the vicious circle of their perpetuation and dissemination. ${ }^{8}$

Therefore, the popular visual culture, frequently assisted by the members of the research community, has conserved an image of science which largely draws its historical references from the period before the $19^{\text {th }}$ century, when science and technology had not yet developed in the current sense. This indicates that there is a strongly consolidated public conception of science that has remained unaffected by the rapid and radical processes of scientific development in the course of the last two centuries. Given that these two centuries were also marked by the improvement of public education, as well as by the diffusion of the visual image as a primary means of communication, one can assume that such social and technological progress had little effect on the formation of the image of scientists. In conclusion, this oldfashioned, stereotypical and inaccurate public image of science is not apt to change. ${ }^{8}$

\section{The context of the study: 'Please draw a researcher'}

The present paper aims at identifying crucial aspects of the image of the scientific researcher in students' drawings. The drawings were produced in the context of 'Researcher's Night $2007^{*}$ ' in three Greek cities: Volos (under the auspices of the University of Thessaly), Patras (under the auspices of the University of Patras), and Heraklion (under the auspices of the Foundation for Research and Technology).

The 2007 'Researcher's Night' focused on the relation between art and research. One of its central actions involved a drawing competition titled "Please draw a researcher", which addressed 8-17 year-old students inviting them to represent visually people of research, as they spontaneously think about them. The 'Researcher's Night 2007' concluded with open events including an exhibition of the drawings created for the competition, the awarding of prizes to the competition winners, lectures, discussions and video-projections. In total, 171 drawings were produced by students (67 boys and 104 girls) in the regions of the three participating Greek institutions. These drawings were scrutinized in order to extract the central aspects of the images of researchers held by Greek adolescents and to compare these images with the ones prevailing in the popular culture, as outlined in the previous section.

More particularly, the present analysis focused on a) the researchers' characteristics considered as indicative of a stereotype model (lab coat, eyeglasses, facial/peculiar hair, research symbols, knowledge symbols, technology products, captions, elements of the natural world) ${ }^{16,20} \mathrm{~b}$ ) on the overall image of the researcher - positive, negative, 'dual', i.e. a combination of the two, or neutral/indeterminate- as depicted in the drawings; c) the gender of the depicted researchers; d) the fields of their specialization; e) the nature of research, that is if it is primarily confined in laboratory settings or not, if it is represented as a practical/manual or a theoretical activity (or a combination of the two), as well as if it is depicted as a solitary or a collaborative endeavour; and f) the elements in the drawings serving as research 'emblems' (for instance test tubes, microscopes, famous researchers etc.).

\section{Facets of students' images of the researcher}

The drawings collected in the course of the 'Please draw a researcher' competition in the scope of the 'Researcher's Night 2007' are undeniably of significant artistic and aesthetic value, especially if one takes into account that they were produced by primary and secondary students. At the same time they

\footnotetext{
* "Researcher's Night" is a European Commission initiative, funded by the Seventh Framework Programme, held at different European cities each year. It aims at bringing science and scientists to the general public. More specifically, this action, with the central catchphrase "Researcher: Someone like us" aims at a) improving the image of the researcher in society; b) raising public awareness about research, innovation and new technologies; c) bridging the gap between researchers and citizens by presenting the possibilities of research and technological foundations to serve society; d) familiarizing youth with techno-scientific issues; e) encouraging teachers and governments to systematically support young people in choosing research careers.
} 
constitute lively and remarkable examples of how Greek students take in, reshape, and reproduce many of the aspects of the dominant popular image of the scientists. This image is abundant with traditional stereotypes and explicit historical references.

What is a 'researcher' for the students who participated to the competition? What is the image they spontaneously allude to about the people of research and how do they choose to represent it? In the following sections the results of the analysis according to the dimensions described in the previous section are presented and discussed. These results are summarised in Table 1.

\begin{tabular}{|c|c|c|c|}
\hline & Boys & Girls & Total \\
\hline \multicolumn{4}{|l|}{ Researcher's characteristics } \\
\hline Lab coat & 6 & 21 & 27 \\
\hline Eyeglasses & 12 & 17 & 29 \\
\hline Facial/peculiar hair & 28 & 29 & 57 \\
\hline Research symbols & 26 & 54 & 80 \\
\hline Knowledge symbols & 14 & 41 & 55 \\
\hline Technology products & 9 & 21 & 30 \\
\hline Captions & 10 & 21 & 31 \\
\hline Elements of the natural world & 27 & 39 & 66 \\
\hline \multicolumn{4}{|l|}{ Overall image of the researcher } \\
\hline Positive & 34 & 66 & 100 \\
\hline Negative & 7 & 6 & 13 \\
\hline 'Dual' & 4 & 5 & 9 \\
\hline Neutral/indeterminate & 22 & 27 & 49 \\
\hline \multicolumn{4}{|l|}{ Researcher's gender } \\
\hline Male & 53 & 56 & 109 \\
\hline Female & 4 & 22 & 26 \\
\hline Male \& Female & 1 & 4 & 5 \\
\hline Absent/indeterminate & 9 & 22 & 31 \\
\hline \multicolumn{4}{|l|}{ Specialization of researchers } \\
\hline Physicists & 3 & 7 & 10 \\
\hline Chemists & 3 & 9 & 12 \\
\hline Biologists & 1 & 9 & 10 \\
\hline Astronomers & 4 & 7 & 11 \\
\hline 'Omniscients' & 12 & 20 & 32 \\
\hline Naturalists & 3 & 4 & 7 \\
\hline Explorers & 19 & 10 & 29 \\
\hline Detectives & 2 & 3 & 5 \\
\hline Other/indeterminate & 20 & 35 & 55 \\
\hline \multicolumn{4}{|l|}{ Research activity } \\
\hline Fieldwork & 52 & 74 & 126 \\
\hline Laboratory work & 15 & 30 & 45 \\
\hline Practical/manual activity & 38 & 43 & 81 \\
\hline Theoretical activity & 0 & 14 & 14 \\
\hline Practical \& theoretical activity & 13 & 24 & 37 \\
\hline Solitary & 52 & 89 & 141 \\
\hline Collaborative & 8 & 7 & 15 \\
\hline \multicolumn{4}{|l|}{ Emblems of research } \\
\hline Test tubes, flasks & 9 & 21 & 30 \\
\hline Microscopes & 4 & 10 & 14 \\
\hline Telescopes & 7 & 9 & 16 \\
\hline Dividers, formulas & 6 & 8 & 14 \\
\hline Spaceships, rockets & 8 & 1 & 9 \\
\hline Molecular models & 2 & 1 & 4 \\
\hline Famous researchers & 9 & 12 & 21 \\
\hline
\end{tabular}

Table 1. Researchers, research activity and emblems in students' drawings. 


\section{The characteristics of the researcher}

Researchers were depicted by students bearing a multitude and a diversity of characteristics, typical of the stereotypic scientist (see Figures 1 and 2). Most frequently the depicted researchers handle research instruments and laboratory equipment like test tubes, microscopes, or telescopes (80 instances), are surrounded by elements of the natural world (animals, plants, or astronomical objects) constituting the object of their study (66 instances), have facial hair (e.g. beard, moustache) and/or peculiar hair-style (57 instances), and use symbols of knowledge such as books, notebooks, blackboards (55 instances). Quite often (in 31 cases) their depictions are accompanied by captions relevant to their research (e.g. mathematical or chemical formulae, taxonomic classifications, captions like "eureka!", etc.), while some researchers (30 instances) are depicted using technological products (mainly computers), wearing eyeglasses (29 cases) and lab coats (29 instances).

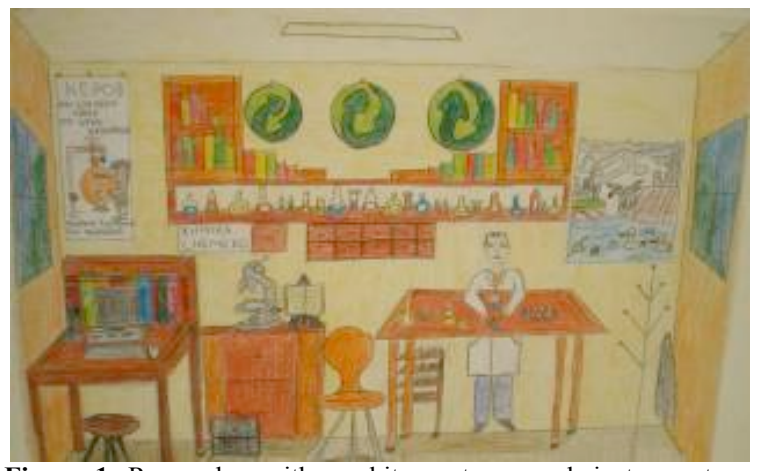

Figure 1. Researcher with a white coat, research instruments, knowledge symbols, technological products, and relevant captions.

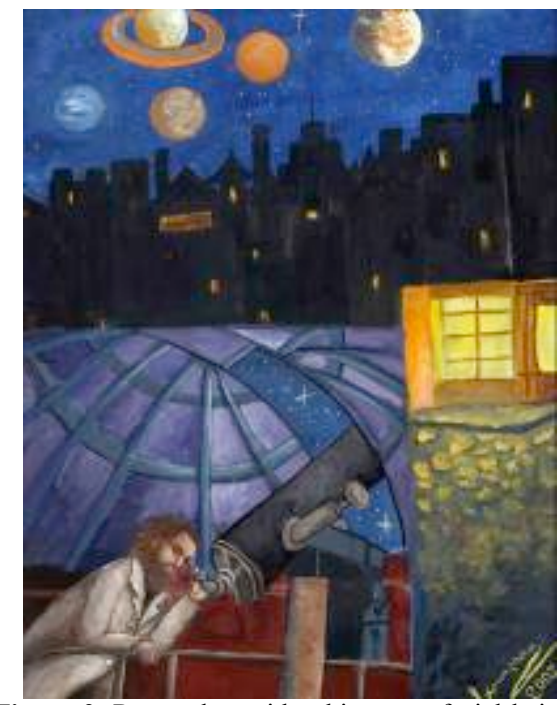

Figure 2. Researcher with white coat, facial hair, research instruments and elements of the natural world.

In a few cases (13 in total) the researcher is presented as an eccentric figure with uncanny (even extraterrestrial) or mythical traits (see Figure 3). Such representations imply a negative image: the researcher seems to be distant, hostile (see Figure 4), possibly disturbed. Some of these drawings inherently involve elements of science fiction (extraterrestrial creatures, or Frankenstein-like figures).

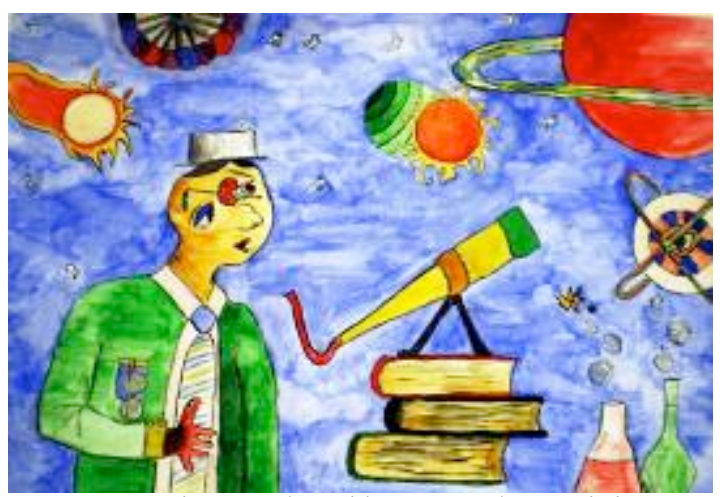

Figure 3. Eccentric researcher with uncanny characteristics.

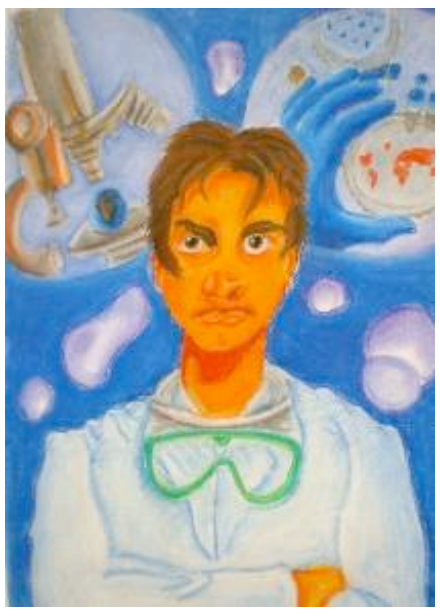

Figure 4. The researcher as a hostile and distant figure. 
However, the vast majority of the drawings (100 instances) reflect a positive image of researchers who observe and study nature motivated by altruism, or by unsaturated curiosity and pure love for the conquest of knowledge. A small number of drawings that belong in this category depict children -or youngsters- in the role of the researcher, who learn by studying books, or observing the world around them. The motive for learning is so strong that in some instances -as in the case of the teenager in Figure 5- the researcher falls asleep during study!

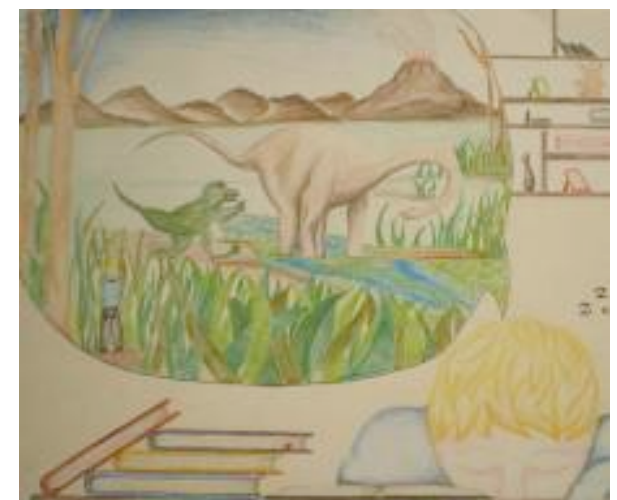

Figure 5. The researcher as a child falling asleep during study.

Some of the drawings ( 9 in total) combine the two aspects of the public image of science and technology, representing a counterpoint between the 'good' and the 'harmful' uses of research, as well as between nature and an artificial environment, created by techno-scientific progress (see Figure 6).

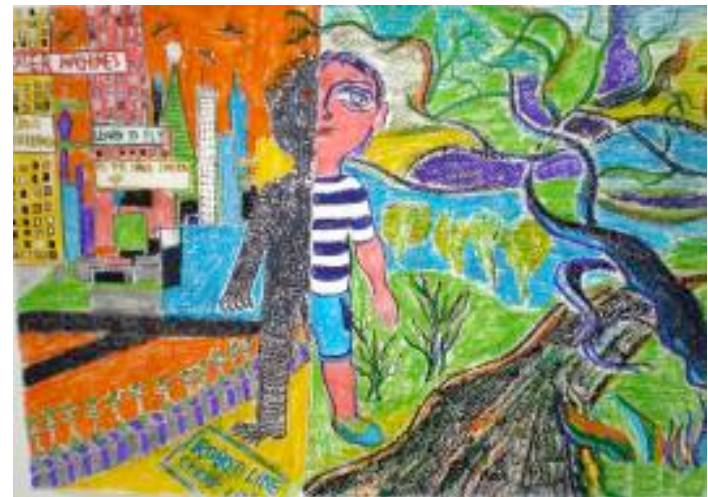

Figure 6. The 'dual' researcher split between the natural, and an ominous man-made, technological environment.

Another central aspect of the stereotypic scientist, reflected in the students' drawings concerns the gender of the represented researcher. The persons drawn by Greek students are largely men (109 instances, see Table 1). Female researchers appear scarcely (26 instances), and are almost exclusively included in girls' drawings. Sometimes (5 instances) female researchers coexist with male ones, either in equivalent roles, or as assistants. ${ }^{10}$ A typical example of such an unequal coexistence is the representation of two researchers in Figure 7: the woman's role is clearly differentiated from that of the man. She is apparently his assistant, dealing with the 'practical' part of the work, while the man, distanced, and holding his notes, is the one who seems to possess and instruct in theoretical knowledge. 


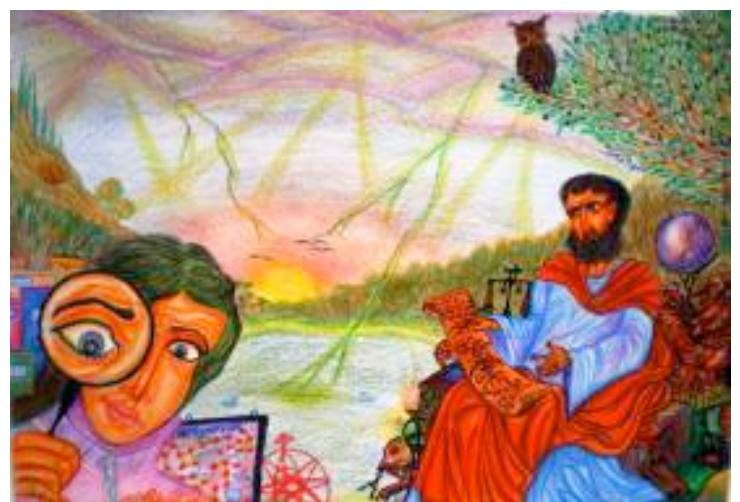

Figure 7. A male and a female researcher: the woman deal with observation, the man with theory.

\section{Fields of specialization and nature of research activity}

The researchers in the students' drawings most often engage in science-related activities. As indicated in table 1, the fields of their expertise involve chemistry (12 instances), astronomy (11 instances), physics (in 10 cases), and biology (10 instances). Occasionally researchers engaged in other fields, like archaeology, are represented. Yet, research fields related with social sciences and humanities generally tend to be absent in the drawings.

In several cases (32 in total) elements from different fields of research activity, corresponding to different science-related disciplines, are combined in the same drawing. The researcher in Figure 8 is an example of such an 'omniscient', with multiple research interests, ranging from mathematics, relativity theory, and 'space science', to chemistry and geography.

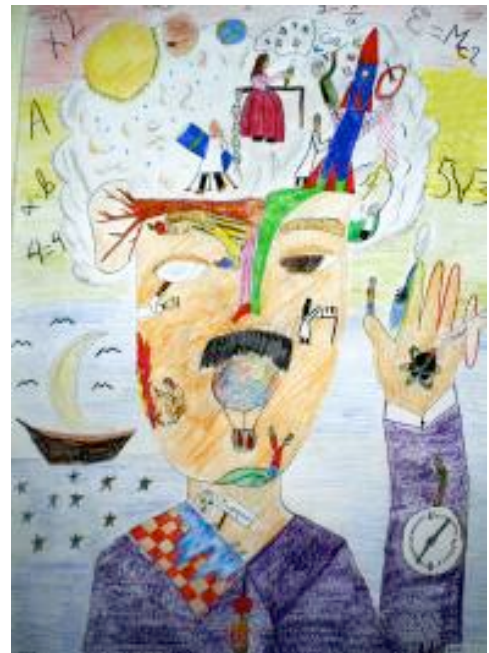

Figure 8. Depiction of multiple elements from different scientific fields.

Overall, the scientific fields represented in the drawings cover wide areas of science, according to a traditional and generalized view. The depiction of modern, specialized fields is generally absent. The only activity with explicit references to current techno-scientific developments, and without traditional origins is space exploration. ${ }^{8}$

Apart from the activities purely related with scientific research, the drawings also include representations of naturalists (7 instances, as in Figure 9), explorers -including great explorers as Magellan and Columbus, as well as space explorers (29 cases), and detectives (5 instances) who, holding magnifying lenses, investigate to solve a mystery. 
There are some differentiations in the types of activities chosen to be represented by boys and girls: male participants tend to depict chemists and biologists more frequently than expected, while females tend to represent researchers as 'omniscients' and explorers.

Moreover, the characteristics of the depicted researchers, as described in the previous paragraph, vary significantly according to the depicted professions. Hence, drawings representing science-related activities include 3.1 elements of the stereotypic scientist (such as research and knowledge symbols, lab coats, eyeglasses etc) on average, while those representing other activities and professions (e.g. explorers, or detectives) include 1.5 such elements on average. Therefore, stereotypic characteristics tend to be related to science and not so much to other fields.

Researchers in the students' drawings are most frequently doing fieldwork (126 instances), observing plants, animals, or the stars (see Figure 9), or exploring space (see Figure 10). Alternatively, they carry out their research in the laboratory (45 instances), using specialized equipment (as in Figure 11).

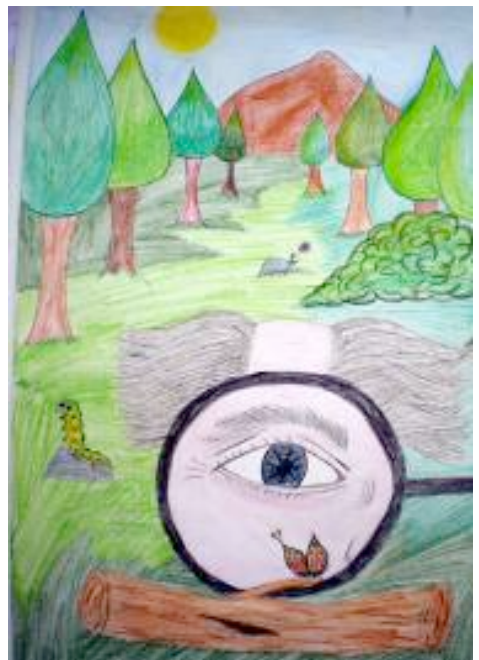

Figure 9. A naturalist researcher observing nature

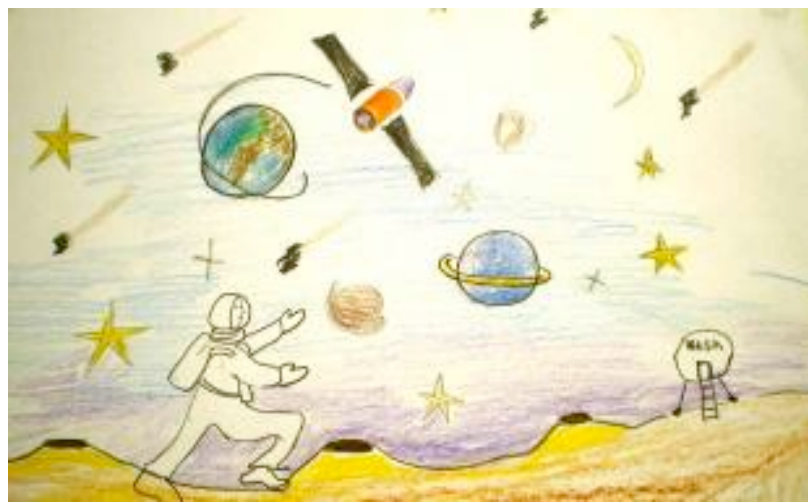

Figure 10. An astronaut exploring space

Another characteristic of the majority of the drawings is that they put forward observation, experimentation and measuring as the central activities in scientific research, while they concentrate much less on study, or on the construction of theoretical models. Research is therefore mainly represented as a manual operation of experimental equipment (81 instances) and secondarily (in only 14 cases) as a theoretical activity. When this happens, theoretical work is largely symbolized by researchers studying books, taking notes, or writing formulas. Moreover, several drawings combine the practical and theoretical aspects of research (37 instances).

Research is mostly considered as a solitary activity (141 instances). The researcher is depicted as working individually, totally concentrated on his/her observations, experimentations, or study. More than one researchers, working in collaboration, appear in only a small number of drawings (15 cases).

\section{The emblems of scientific research}

The students' drawings use the popular visual culture symbolisms widely to represent scientific research. These symbolisms refer to a variety of science 'emblems' [8], ranging from traditional research and observation instruments, like test tubes and glass flasks for chemistry (30 instances, see Figure 11), microscopes for bio-medicine (14 instances), telescopes for astronomy (16 instances), and dividers or formulas for mathematics (14 cases). Spaceships and rockets are depicted as emblems of space exploration (14 instances). In a few cases (4 in total) molecular models (like the DNA molecule in Figure 12) play the role of research emblems. 


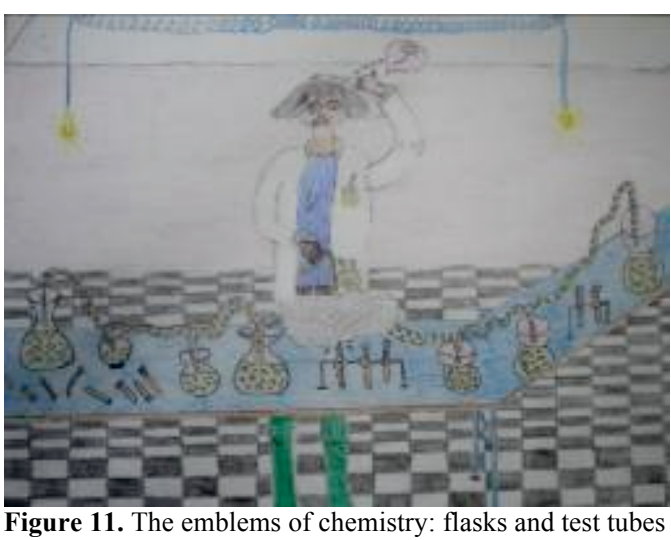

In several drawings -especially in those related to physics- the role of emblems is taken up by famous scientists (21 instances) who have put their mark on the development of their discipline instead of laboratory equipment. Such emblematic figures include Einstein (as in Figure 13), Newton, Torricelli,

In several drawings -es
scientists $(21$ instances $)$
laboratory equipment. S
Archimedes, and others.

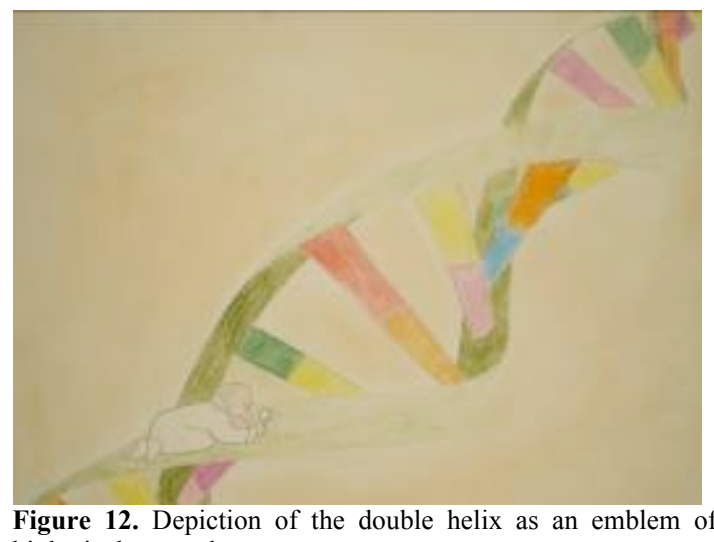

Figure 12. Depiction
biological research

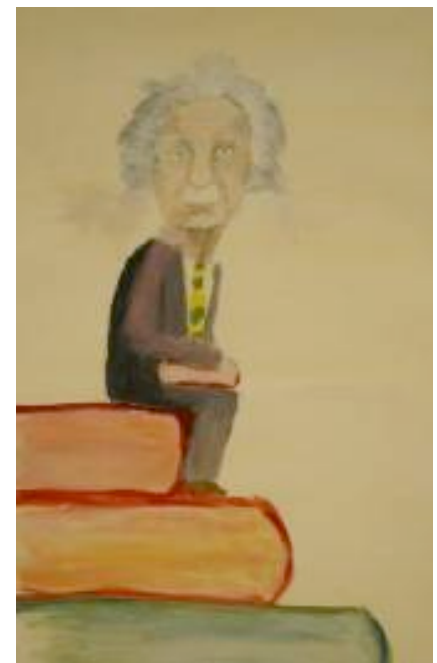

Figure 13. Einstein as an emblematic figure of scientific research.

Chemistry emblems (i.e. test tubes and flasks) are depicted in girls' more frequently than in boys' drawings, while the opposite trend is observed in the case of spaceships and rockets (emblems of space exploration), which are almost exclusively introduced in boys' drawings.

Another characteristic of the drawings is that laboratory equipment - a typical symbol of research activity - is generally traditional (like the test tubes, flasks, telescopes, dividers, and microscopes, already mentioned). Modern experimental settings with specialized or complex technological equipment and references to contemporary research methods are not frequently depicted.

\section{Discussion and conclusion}

The preceding analysis verifies to a great extent previous research outcomes related to the image of scientists. In particular, the characteristics attributed to researchers (research symbols, lab coats, eyeglasses etc) are very similar to those recorded internationally with only minor differentiations across decades. ${ }^{19,20,21}$ Such characteristics have also been recorded in different samples of Greek students ${ }^{26,33,34}$ as well as teachers. ${ }^{29,30}$ 
Similarly, as previously observed in a multitude of studies, ${ }^{4,16,20,28,29,30}$ the present one reveals a robust gender stereotype related to scientific researchers, since the vast majority of depicted persons were male. This outcome is particularly important, especially if one takes into account that girl participants were overrepresented in the competition in comparison to boys. Therefore the fact that the girls -exactly like boys- spontaneously tend to draw male researchers indicates that they barely identify themselves with the role of a dynamic researcher, an attitude which will possibly deter them from engaging in scientific research in the future.

As is the case for other characteristics of the drawings, they represent in majority a fairly restricted and rather out-dated image of scientific research as a primarily practical or manual activity. ${ }^{35}$ This is perhaps due to the fact that the theoretical aspect of research - and theory construction in particular- is difficult to represent graphically. Also, scientific research is mainly depicted as solitary, while contemporary technoscientific progress presupposes teamwork, communication and collaboration between researchers. ${ }^{8}$ This feature of the drawings might be methodologically attributed to the instruction given to the students participating to the drawing competition ("Please draw a researcher"), which possibly directed them towards drawing a single person.

Furthermore, prevalent 'emblems' of scientific research reflected in popular and self-images of science ${ }^{8}$ have been widely used by the students in this study. Such emblems mainly involve research instruments, as well as mathematic formulas, or molecular models used to designate different areas of research. The emblematic objects represented in students' drawings have been invented since the $17^{\text {th }}$ century, or even earlier. The most common exemption are rockets, which constitute a modern emblem corresponding to 'space exploration', which is not a traditional discipline, but a modern field of techno-scientific research. In other cases (especially regarding the discipline of physics) scientific research is identified with the portrait of an eminent historical person.

Despite the major similarities of the results presented in this study with the ones reported by previous ones, some noteworthy differences were also traced. These are firstly related to the setting of scientific research, which according to others is vastly confined in research laboratories ${ }^{1,19,20}$ while the majority of the drawings analyzed in the present context depict researchers working outdoors, performing fieldwork. Further investigation is required to determine if this differentiation is related to the context of production of the drawings ("Draw a researcher" instead of "Draw a scientist" in previous studies), or should be attributed to other factors.

Secondly, some interesting differentiations emerge in regards to the types of activity recorded. In the context of previous studies, using the 'Draw A Scientist Test' technique ${ }^{20}$ depictions of medical doctors, engineers, and especially teachers were quite common. ${ }^{5,15,16}$ In the context of the present study these professions are generally absent (with the exemption of 3 drawings depicting medical doctors). Instead, other types of research activity emerge, uncommon in studies concerning the image of scientists. These involve explorers, detectives, but especially 'omniscient' figures, promoted by several drawings and originating historically from the outset of science, which evidently does not correspond to its contemporary constitution in finely specialized and restricted research areas. ${ }^{8,10}$

Last, but not least, a remarkable outcome of the present study concerns the dominating positive image of researchers emanating from the students' drawings, also identified in recent studies, ${ }^{35}$ which employed different research techniques from the present. This generally positive image diverges from the dominating trends in the popular and public imagery of scientists, who are generally portrayed as eccentric, unsocial, unfriendly, and/or unpleasant figures. Such depictions - rather infrequent in the present study - are connected to the fear and awe created by techno-scientific research, which is considered as an endeavor that can put humanity in danger, ${ }^{7,10,11}$ with elements of science fiction, an outcome typically observed in students' conceptions of scientists internationally. ${ }^{17,18,19}$ The question deriving from this differentiation is whether it is characteristic of the specific -and restricted- sample of students, or it has been induced by the context of production of the specific drawings. In other words, inviting people to draw scientists might yield less positive images than when inviting them to draw researchers. This question also requires further exploration.

In conclusion, the image of the researcher remains for the majority of young people intensely stereotypic and particularly traditional, mainly inspired from archetypes formed centuries ago. Youngsters, like previous generations, espousing a primarily romantic image of the researcher as a naturalist, do not seem adequately familiar with the organisation, specialisation, possibilities, means, and achievements of modern research, or with its relations with society. ${ }^{32}$ Instead, young people draw their 
images about researchers from outdated and distorted clichés about science and technology, which originate from popular culture and fiction. Such clichés, in their negative version, may also embody the fear of distress possibly caused by science and technology on society and the planet. The students' drawings, especially remarkable and enlightening in all respects, indicate the necessity for a more targeted and effective communication between research institutions and society.

Sometimes scientific researchers wonder how they could render their public image more 'realistic'. The question itself verifies that their self-image does not coincide with the public one. It also means that scientists have little effect on the selection and production of the images of their own profession. They are mere observers of a mythology created by the popularization of science, which is not amenable to any control.

So far, the efforts of formal education as well as those of informal channels of science diffusion (like the mass media, cinema, documentary films, museums and science centers) have not proven particularly effective in promoting more adequate and realistic images of scientific research, if one takes into account the stability of the stereotypes described in the previous sections. A key solution to this problem could be the involvement of the research community in a systematic promotion of its people and their activities to the general public, aiming at refuting obsolete and deceiving notions. It is therefore apparent that universities and other research institutions face a significant challenge: to present their research activity in a comprehensible and attractive manner, to open their gates to the public, to communicate with society, which - after all - funds them and ensures their viability.

Therefore, contemporary scientific research has yet a long way to cover in order to restore its public image and start influencing the relevant conceptions and attitudes of the public and especially those of young people as future citizens and potential researchers.

\section{Notes and references}

1 C.R. Boylan et al. (1992), Beyond stereotypes, Science Education 76: 465-476.

2 V. Christidou (2006), Greek Students' Science-Related Interests and Experiences: Gender Differences and Correlations, International Journal of Science Education 28: 1181-1199.

3 C. Dawson (2000), Upper primary boys' and girls' interests in science: have they changed since 1980?, International Journal of Science Education 22: 557-570.

4 K.D. Finson (2002), Drawing a scientist: What we do and do not know after fifty years of drawings, School Science and Mathematics 102: 335-345.

5 Y.Y.H. Fung (2002), A comparative study of Primary and Secondary School Students' Images of Scientists, Research in Science and Technological Education 20: 199-213.

6 R. Schibeci and L. Lee (2003), Portrayals of Science and Scientists, and 'Science for Citizenship', Research in Science and Technological Education 21: 177-192.

7 J. Song and K.S. Kim (1999), How Korean students see scientists: the images of the scientist, International Journal of Science Education 21: 957-977.

8 J. Schummer and T.I. Spector (2008), Popular images versus self-images of science, in B. Hüppauf and P. Weingart (eds), Science Images and Popular images of the Sciences, Routledge, N.Y.

9 B. Hüppauf and P. Weingart (2008), Images in and of Science, in B. Hüppauf and P. Weingart (eds), Science Images and Popular images of the Sciences, Routledge, N.Y.

10 E. Flicker (2008), Women scientists in mainstream film, in B. Hüppauf and P. Weingart (eds), Science Images and Popular images of the Sciences, Routledge, N.Y.

11 P. Pansegrau (2008), Stereotypes and images of scientists in fiction films, in B. Hüppauf and P. Weingart (eds), Science Images and Popular images of the Sciences, Routledge, N.Y.

12 P. Weingart (2008), The ambivalence towards New Knowledge: Science in Fiction Film, in B. Hüppauf and P. Weingart (eds), Science Images and Popular images of the Sciences, Routledge, N.Y.

13 J. Steinke (2005), Cultural representations of gender and science: Portrayals of female scientists and engineers in popular films, Science Communication 27(1): 27-63.

14 W.J.T. Mitchell (2008), Image Science, in B. Hüppauf and P. Weingart, Science Images and Popular images of the Sciences, Routledge, N.Y.

15 E. Rubin, V. Bar and A. Cohen (2003), The images of Scientists and Science among Hebrew-and Arabic-speaking pre-service teachers in Israel, International Journal of Science Education 25: 821-846.

16 H.C. She (1998), Gender and grade level differences in Taiwan students' stereotypes of science and scientists, Research in Science and Technological Education 16: 125-135. 
17 G. Aikenhead (1988), An analysis of four ways of assessing student beliefs about STS topics, Journal of Research in Science Teaching 25(8): 607-629.

18 R. Driver et al. (1996), Young People's Images of Science, Open University Press, Buckingham.

19 M. Mead and R. Metraux (1957), The image of the scientist among high school students: A pilot study, Science 126: 384-390.

20 D.W. Chambers (1983), Stereotypic images of the scientist: The Draw-a-Scientist Test, Science Education 6: 255-265.

21 H.C. She (1995), Elementary and middle school students' image of science and scientists related to current science textbooks in Taiwan, Journal of Science Education and Technology 4: 283-294.

22 C.L. Mason, J.B. Kahle and A.L. Gardner (1991), Draw-A-Scientist Test: Future implications, School Science and Mathematics 91: 193-198.

23 L.D. Newton and P.D. Newton (1998), Primary children's conceptions of science and the scientist: Is the impact of a National Curriculum breaking down the stereotype?, International Journal of Science Education 20: 1137-1149.

24 D.B. Rosenthal (1993), Images of scientists: A comparison of biology and liberal studies majors, School Science and Mathematics 93: 212-216.

25 R.A. Schibeci and J.P. Riley (1986), Influence of students' background and perceptions on science attitudes and achievement, Journal of Research in Science Teaching 23: 177-187.

26 V. Christidou, F. Bonoti, and Z. Anastasiou (2006), How do primary school children represent the male and female scientist?, In: E. Stavridou (ed.), Science Education: Methods and Learning Technologies - Proceedings of the $3^{\text {rd }}$ Panhellenic Conference of the Association for Science Education (EDIFE). New Technologies Editions, Athens [In Greek].

27 A. Mays (2001), Student stereotypes of scientists: Can they be changed?, Retrieved June 5, 2006; available at http://www.bamaed.ua.edu/ amays/ actionresearch.htm.

28 W.J. Sumrall (1995), Reasons for the perceived images of scientists by race and gender of students in grades 1-7, School Science and Mathematics 95: 83-90.

29 V. Hatzinikita (2007), Images of the scientist held by educators in Greece, International Journal of Learning 14(7): 145-154.

30 V. Hatzinikita, V. Christidou and F. Bonoti (2009), Teachers' pictorial representations of the scientist, in A. Selkirk and M. Tichenor (eds.), Teacher Education: Policy, Practice and Research. Nova Science Publishers, Hauppauge N.Y.

31 I.N. Quita (2003), What is a scientist? Perspectives of Teachers of Color, Multicultural Education Fall, pg. 29-31.

32 P. Rodari (2007), Science and scientists in the drawings of European children, JCOM 06(03): C04.

33 K. Polizou (2009), Secondary students' representations of the scientist and his/her activity: exploration of determinant factors and teaching implications, Unpublished Masters Dissertation, Patras (Greece): Hellenic Open University, [In Greek].

34 G. Samaras (2009), Primary pupils' representations of the scientist: exploration of determinant factors, Unpublished Masters Dissertation, Volos (Greece): University of Thessaly, [In Greek].

35 D. Gouthier (2007), Teachers' perception of the European scientists, JCOM 06:(03): C06.

\section{Author}

Dr. Vasilia Christidou is Associate Professor at the Department of Preschool Education, University of Thessaly, Greece. Her current research interests include teaching and learning in science, the promotion of public engagement in science issues, and the process of recontextualization of scientific texts addressed to non-experts. She has coordinated and participated in a number of research projects, and has published numerous research articles and studies in journals, books, and conference proceedings related to public engagement in science issues and science communication. E-mail: vchristi@ece.uth.gr.

How TO CITE: $\quad$ V. Christidou, Greek students' images of scientific researchers, Jcom 09(03) (2010) A01. 